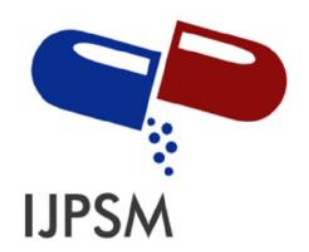

Shaikh Bilal J. et al, Int. Journal of Pharmaceutical Sciences and Medicine (IJPSM),

Vol.6 Issue. 6, June- 2021, pg. 1-14

ISSN: 2519-9889

Impact Factor: $\mathbf{3 . 4 2 6}$

\title{
NANOSPONGES: AN EVOLUTIONARY TREND FOR TARGETED DRUG DELIVERY
}

\section{Shaikh Bilal J."; Patil Abhishek S.; Bhosale Ankush S.; Raut Indrayani D.; Nitalikar Manojkumar M.}

Rajarambapu College of Pharmacy, Kasegaon (MS) India

Email id- shaikhbillu7@gmail.com

DOI: 10.47760/ijpsm.2021.v06i06.001

\author{
Correspondence Address: \\ Dr. Indrayani D. Raut \\ Assistant Professor, \\ Rajarambapu College of Pharmacy, Kasegaon (MS) India \\ Email id: idraut7363@gmail.com \\ Contact no. - 8600009718
}

\begin{abstract}
Recent developments in nanotechnology have made the framework for the growth of new nanoscale biomaterials with numerous potential uses in nanomedicine. The nanosponge is a new and emerging technology that can be used to target medicine delivery in a precise and regulated manner. Nanosponge is an important component of advanced medication delivery. It is a specialized assisting system for regulated medication delivery of both lipophilic and hydrophilic medicines in a regulated manner. Effective medication delivery at a specific location allowed for more exact control of release rates and boosted health-care system compliance, but the chemistry having complex shape complicated things. The invention of nanosponges, on the other hand, has provided significant approach to tackling this problem. The technology of Nanosponge has been studied widely for the delivery of drugs for oral, topical, and parental administration. Enzymes, proteins, vaccines, and antibodies can all be delivered effectively using nanosponges. It is more effective for targeted drug delivery systems because it improves solubility, bioavailability, and decreases side effects by releasing the drug at specific site. It has the ability to improve drug solubility and can be manufactured into oral, topical, and parenteral dose forms. This type of carrier system is best for drugs with low bioavailability. Nanosponges can contain both lipophilic and hydrophilic medicines. Nanosponges are extremely permeable, with a high proclivity for ensuring dynamic particles and programmable delivery. This review article discusses nanosponges in general, their advantages \& disadvantages, mechanism; factors influence nanosponge, preparation methods, evaluation parameters as well as applications.
\end{abstract}

Keywords: Nanosponge, targeted site, solubility, mechanism, applications. 


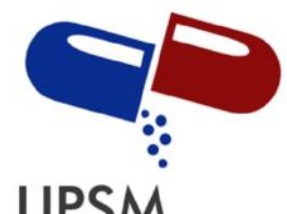

Shaikh Bilal J. et al, Int. Journal of Pharmaceutical Sciences and Medicine (IJPSM), Vol.6 Issue. 6, June- 2021, pg. 1-14

ISSN: 2519-9889

Impact Factor: 3.426

\section{Introduction $^{[1][3][7]}$}

Targeting drug delivery systems to achieve the desired result has been a long-term goal. Nanosponge drug delivery systems were originally only available as a topical administration system, but in the $21^{\text {st }}$ century, Nanosponges can now be administered orally as well as intravenously (IV). Nanosponge is a new type of material made composed of very small particles having a very narrow cavity of a few nanometers. These little spaces can be filled with a variety of materials. These tiny particles have the capacity to convey both hydrophilic and lipophilic drug substances, as well as enhance the stability of pharmacological substances or compounds that are weakly water soluble. The nanosponges are a 3D polyester scaffold (backbone) or network that can degrade spontaneously. Nanosponges are made by combining these polyesters with a cross linker in a solution. Because polyester is normally biodegradable, it breaks down slowly in the body. When the scaffold of nanosponges degrades, it releases the drug molecules that have been loaded in an unfavorable manner.

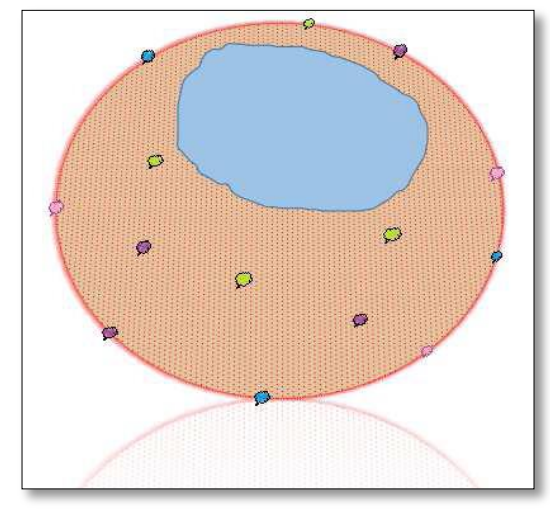

Figure 1: A nanosponge structure with a cavity for drug loading.

The nanoparticles can be categorized into three groups based on how they associate with medicines.

Encapsulating Nanoparticles: Nanosponges and nano capsules are examples of encapsulating nanoparticles. Alginate Nanosponges, which are sponge-like nanoparticles, have multiple hollows that allow drug molecules to pass through. Nanoparticles are also encapsulated in nano capsules such as poly (isobutyl cyanoacrylate) (IBCA). Drug molecules can be located in their aqueous core.

Complexion Nanoparticles: This category comprises compound nanoparticles that use electric charges to attract molecules.

Conjugating Nanoparticles: These nanoparticle cominations link or connect to drug substance with covalent bond. 


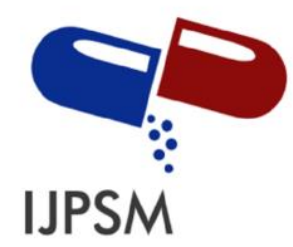

\section{Shaikh Bilal J. et al, Int. Journal of Pharmaceutical Sciences and Medicine (IJPSM),} Vol.6 Issue. 6, June- 2021, pg. 1-14

ISSN: 2519-9889

Impact Factor: 3.426

\section{Advantages ${ }^{[1][2][3][5][13][14]}$}

1. They act as a self-sterilizer even though their average pore size is $0.25 \mu \mathrm{m}$, in which bacteria cannot penetrate.

2. This method allows for the trapping of chemicals and the reduction of side effects along with improved stability, elegance, and formulation flexibility.

3. Nanosponges are non-irritating, non-mutagenic, non-allergic, and non-toxic.

4. Allows for the incorporation of immiscible liquids, which improves material processing by converting liquids to powders.

5. Commercial production is easy to scale up.

6. Nanosponges can be regenerated using eco-compatible solvents, inert hot gases, mild heating, and changing strength.

7. Nanosponges aid in the removal of harmful and venomous substances from the body and minimize side effects.

8. Nanosponges can deliver drug molecules in a controlled fashion.

9. Nanosponges complexes are stable throughout a wide $\mathrm{pH}$ range (from 1 to 11 ) and at $130^{\circ} \mathrm{C}$.

10. It can help to mask the drug's unpleasant taste.

11. Improves the solubility of poorly soluble drugs, resulting in improved in bioavailability.

12. The drug is resistant to deterioration.

13. It has an extended release condition that allows for continued action for up to 12 hours.

\section{Disadvantages ${ }^{[1][2][3][5][13][14]}$}

1. Nanosponges can be paracrystalline or crystalline in nature.

2. The degree of crystallization determines the loading capacity of nanosponges.

3. Different loading capabilities can be found in paracrystalline nanosponges.

4. Nanosponges can only hold encapsulated molecules and are therefore unsuitable for bigger molecules.

5. Dose dumping is a possibility.

6. Nanosponges can be semi-translucent or have a glassy appearance.

7. The stacking limit of nanosponges is mostly determined by the degree of crystallization.

\section{Mechanism of drug release from nanosponges ${ }^{[1][14]}$}

Because of nanosponges particles have an open structure; the active constituent is free to move in and out of the particles into the vehicle until the equilibrium is reached when the vehicle becomes saturated. When the product is applied to the skin, the active constituent already present in the vehicle becomes unsaturated, disrupting the balance. This will initiate the flow of active substance from the nanosponge's particle into the vehicle, which will then be 


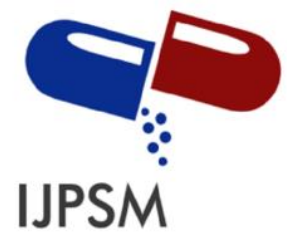

Shaikh Bilal J. et al, Int. Journal of Pharmaceutical Sciences and Medicine (IJPSM), Vol.6 Issue. 6, June- 2021, pg. 1-14

ISSN: 2519-9889

Impact Factor: 3.426

applied to the skin until the vehicle has dried or been absorbed. The sponge particle matter that remains on the skin surface (Stratum Corneum) will continue to distribute the active ingredient to the skin even after the vehicle has dried. As a result, the action of the release is prolonged. Even after that, nanosponges particles maintained on the stratum corneum's surface will progressively release active substance to the skin, resulting in a protracted release.

\section{Preparation methods of nanosponges ${ }^{[8]}$}

The following methods are designed to prepare Nanosponges:

1. Melt method

2. Solvent diffusion methods

a) Emulsion solvent diffusion method

b) Quasi - emulsion solvent diffusion

\section{Solvent Method}

4. Ultrasound Assisted Method

5. Polymerization

\section{Melt method $^{[8]}$}

In melt method, cyclodextrin reacts with a suitable crosslinker such as dimethyl carbonate, diphenyl carbonate, isocyanates, diaryl carbonates, carbonyldiimidazole (C7H6N40), carboxylic acid anhydrides, and 2, 2-bis (acrylamide) acetic acid. All of the ingredients are carefully integrated and placed in a $250 \mathrm{~mL}$ flask heated to $100^{\circ} \mathrm{C}$ for 5 hours with a magnetic stirrer. The mixture is allowed to cool before the resulting product is broken down and rinsed with a suitable solvent to remove any unreacted excipients.

\section{Solvent diffusion methods}

\section{a) Emulsion solvent diffusion method ${ }^{[8]}$}

Two different levels of organic and aqueous phases are used in this technique. Drug and polymer are combined in the organic phase, whereas polyvinyl alcohol (PVA) is employed in the aqueous phase. After dissolving the medication and polymer in the appropriate organic solvent, the aqueous phase is slowly mixed in and agitated for 2 or more hours at $1000 \mathrm{rpm}$ using a magnetic stirrer. The ready nanosponges are next filtered, rinsed, and dried in the air at room temperature or in a vacuum oven at $40^{\circ} \mathrm{C}$ for 24 hours.

b) Quasi - emulsion solvent diffusion ${ }^{[2]}$

The nanosponges can also be prepared by Quasi-emulsion solvent diffusion method with various polymer concentrations. The inner phase was made by dissolving eudragit RS100 in a suitable solvent. The drug can then be added to the solution and dissolved at $350^{\circ} \mathrm{C}$ employing ultrasonication. The inner phase was placed into the waterbased PVA solution (outer phase) and stirred for 1 hour before filtering to separate the nanosponges. The nanosponges are dried for 12 hours in an air-heated oven at $40^{\circ} \mathrm{C}$. 


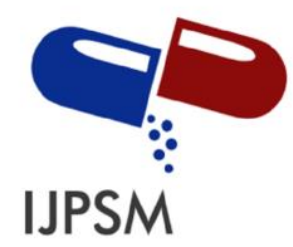

Shaikh Bilal J. et al, Int. Journal of Pharmaceutical Sciences and Medicine (IJPSM), Vol.6 Issue. 6, June- 2021, pg. 1-14

ISSN: 2519-9889

Impact Factor: 3.426

\section{Solvent Method ${ }^{[8]}$}

In this method, a polar aprotic solvent, such as dimethyl formamide or dimethyl sulfoxide, is combined with a suitable polymer. Then, in a molar proportion of $4-16$, this blend is blended with a large amount of crosslinker. The reaction is given at temperatures ranging from $10^{\circ} \mathrm{C}$ to the solvent's reflux temperature for periods ranging from 1 to 48 hours. Carbonyl compounds such as dimethyl carbonate and carbonyl diimidazole (C7H6N4O) are popular crosslinkers. On completion of the reaction, the mixture is allowed to cool at room temperature, after which the chemical is combined with an excess of distilled water, improved by percolation under vacuum, and instantly purified by long-term Soxhlet extraction with ethanol, and finally dried off under vacuum. The dry product is ground in a mechanical mill to make a fine powder.

\section{Ultrasound Assisted Method ${ }^{[1]}$}

Nanosponge is made by reacting polymers with cross-linkers in the absence of solvent and under sonication in this process. This approach will produce spherical and uniformly sized nanosponges. At a flask, combine the polymer and cross-linker in a specific molar ratio. Heat the flask to $900^{\circ} \mathrm{c}$ in an ultrasonic bath filled with water. For 5 hours, sonicate the mixture. Allow the mixture to cool before breaking the result into pieces. Rinse the product with water to remove the non-reacted polymer, then purify with ethanol after a longer soxhlet extraction. Dry the finished product under vacuum and keep it at $250^{\circ} \mathrm{C}$ until needed.

\section{Polymerization ${ }^{[2]}$}

In the monomer, a non-polar drug solution is prepared, to which an aqueous phase is added, usually incorporating surfactant and dispersant to enhance suspension. Once the suspension with discrete droplets of the required size has been created, the monomers are activated either by catalysis or by increasing the temperature. The polymerization process results in the construction of a reservoir system with pores that open at the surface.

\section{Factors influence Nano sponge formation}

\section{Type of polymer ${ }^{[1][2]}$}

The type of polymer used can have an impact on the creation and performance of Nanosponges. The cavity size of a nanosponge should be large enough to admit a drug molecule of a specific size for complexation. The type of polymer employed in nanosponges can have an impact on their formulation and performance. A cross-linker polymer with high efficiency turns molecular nanocavities into a 3D nanoporous structure

a) Hydrophilic Nanosponge: Epichlorohydrin is used as a cross linker to create hydrophilic nanosponge. Even in immediate release formulations, hydrophilic nanosponges can change the pace of drug release and improve drug absorption across biological barriers, making them an effective drug carrier. 


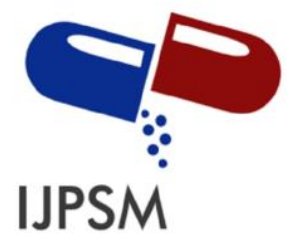

Shaikh Bilal J. et al, Int. Journal of Pharmaceutical Sciences and Medicine (IJPSM), Vol.6 Issue. 6, June- 2021, pg. 1-14

ISSN: 2519-9889

Impact Factor: 3.426

b) Hydrophobic Nanosponge: As a crosslinker, diphenyl carbonate, pyromellitic anhydride, diisocynates, and carbonyl diimidazole can be used to prepare hydrophobic nanosponges. They act as sustained release carriers for water-soluble drugs such as peptides and proteins.

\section{Type of drugs and Medium Used for Interaction ${ }^{[2]}$}

Certain properties of drug compounds that will be complexed with nanosponges are listed below.

a) Molecules with a molecular weight of 100 to 400 Daltons

b) The drug molecule is made up of no more than five condensed rings.

c) Water solubility is less than $10 \mathrm{mg} / \mathrm{ml}$.

d) The substance's melting point is less than $250^{\circ} \mathrm{C}$.

\section{Temperature ${ }^{[1]}$}

Drug/Nanosponge complexation is influenced by temperature changes. The magnitude of the apparent stability constant of the Drug/Nanosponge complex decreases with increasing temperature, which could be due to a reduction in drug/nanosponge interaction forces such as van der Waal forces and hydrophobic forces. Because the drug/nanosponge interaction forces are reduced as temperature rises, the apparent stability constant decreases in magnitude. As a result, when making nanosponges, it's critical to keep the temperature under strict control.

\section{Method of preparation ${ }^{[1]}$}

Drug/Nanosponge complexation can be affected by how the drug is loaded into the nanosponge. However, because the success of a method is dependent on the nature of the drug and polymer, freeze drying has been proven to be the most successful approach for drug complexation in many circumstances.

\section{Degree of substitution ${ }^{[5]}$}

The type, number, and position of the substituent on the parent molecule may have a significant impact on the nanosponge's capacity to complex. There is a correlation between the number of substitutions present and the degree of crosslinking; the more substituents present, the greater the possibility of higher crosslinking. Due to additional linkages between polymers forming a mesh type network, higher degrees of crosslinking will give highly porous nanosponges. The position of substitution is determined by the conditions of production. Due to the occupancy of a different position by the functional group on the parent molecule, a change in the manufacturing method will result in materials with different physicochemical properties. 


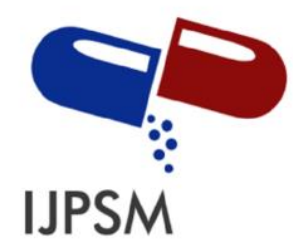

Shaikh Bilal J. et al, Int. Journal of Pharmaceutical Sciences and Medicine (IJPSM), Vol.6 Issue. 6, June- 2021, pg. 1-14

ISSN: 2519-9889

Impact Factor: 3.426

\section{Evaluation Parameters of Nanosponges ${ }^{[1]}$}

\section{Particle size and polydispersity index}

Dynamic light scattering, using a 90 Plus particle sizer with MAS OPTION particle sizing software, or laser light diffractometry, using a Malvern Zetasizer can be used to estimate particle size. The mean diameter and polydispersity index can be calculated using this information.

\section{Morphology and surface topography}

By coating the drug, nanosponges, and the product (drug/nanosponge complex) with gold-palladium under an argon environment at room temperature, scanning electron microscopy (SEM) and transmission electron microscopy (TEM) can be used to analyze the morphology and surface topography of the drug, nanosponges, and the product (drug/nanosponge complex). The production of inclusion complexes is shown by the difference in crystallization states of the raw materials and the finished product as observed under an electron microscope.

\section{Thermoanalytical methods}

Before the nanosponge is thermally destroyed, thermoanalytical procedures are used to see if the drug substance changes. Drug substance alterations include melting, evaporation, disintegration, oxidation, and polymorphic transitions. The formation of a complex is implied by the presence of a change in the drug substance. The thermogram produced by DTA and DSC can identify broadening, shifting, the formation of new peaks, and the elimination of certain peaks. Weight loss changes can also be employed to support the formation of inclusion complexes.

\section{Single crystal $X$-ray structural analysis and $X$-ray diffractometry}

Inclusion complexation in the solid state can be detected using powder X-ray diffractometry. The crystalline character of the drug is altered by the complicated synthesis of the drug in nanosponges, which modifies the diffraction patterns. The complicated creation causes existing peaks to sharpen, the creation of a few new peaks, and the shifting of some summits. The complex development is indicated by the variation in diffraction patterns are useful in determining the chemical decomposition and complicated development of a mixture of chemicals. The exact inclusion structure and manner of interaction can be determined via single crystal X-ray structural analysis. The interaction between the host and guest molecules can be identified, as well as the exact geometrical relationship.

\section{Infra-Red spectroscopy}

The interaction between nanosponges and drug molecules in the solid state is quantified using infrared spectroscopy. When the fraction of the guest molecules encapsulated in the complex is less than $25 \%$, bands that may be assigned to the included part of the guest molecules are easily obscured by the bands of the 


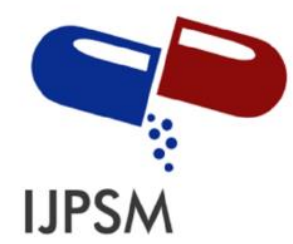

Shaikh Bilal J. et al, Int. Journal of Pharmaceutical Sciences and Medicine (IJPSM), Vol.6 Issue. 6, June- 2021, pg. 1-14

ISSN: 2519-9889

Impact Factor: 3.426

nanosponge spectrum. The technique is less clarifying than other techniques and is not generally suitable for detecting inclusion complexes.

\section{Thin Layer Chromatography}

The Rf values of a drug molecule decrease significantly in Thin Layer Chromatography, which assists in detecting the complex formation between the drug and nanosponge.

\section{Loading efficiency and production yield}

The following equation can be used to compute the loading efficiency $(\%)$ of nanosponges.

$$
\mathbf{L E}=\frac{\text { Actual drug content in nanosponges }}{\text { Theoretical drug content }} \times 100
$$

After determining the exact start weight of the raw materials and the final weight of the nanosponge obtained, the following equation can be used to compute the production yield of nanosponges.

$$
\text { Production yield }=\frac{\text { Practical mass of nanosponge }}{\text { Theoretical mass }(\text { drug }+ \text { polymer })} \times 100
$$

\section{Resiliency}

Sponge resiliency (viscoelastic characteristics) can be altered to generate softer or firmer beadlets depending on the final formulation's requirements. The rate of release is slowed by increased crosslinking. As a result, the resiliency of sponges will be investigated and optimized in accordance with the requirements, taking into account release as a function of cross-linking with time.

\section{Solubility studies}

The phase solubility method proposed by Higuchi and Connors, which evaluates the effect of a nanosponge on drug solubility, is the most commonly used method for studying inclusion complexation. The degree of complexation is indicated by phase solubility diagrams.

\section{Zeta potential ${ }^{[2]}$}

Surface charge is measured by the zeta potential. An additional electrode in the particle size equipment can be used to measure it. Nanosponges containing materials were extracted and diluted with $0.1 \mathrm{~mol} / \mathrm{KCl}$ before being placed in an electrophoretic cell with a $15 \mathrm{~V} / \mathrm{cm}$ electric field applied. After averaging the complete measurement, the mean hydrodynamic diameter and poly dispersity index were computed.

\section{Dissolution test}

The dissolution profile of nanosponges can be analyzed using a modified USP XXIII dissolving equipment with a $5 \mathrm{~m}$ stainless steel mesh basket rotating at $150 \mathrm{rpm}$. To achieve sink conditions, the dissolution 


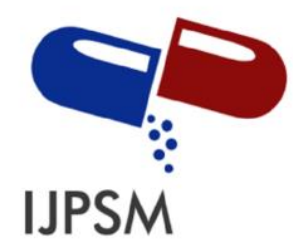

Shaikh Bilal J. et al, Int. Journal of Pharmaceutical Sciences and Medicine (IJPSM), Vol.6 Issue. 6, June- 2021, pg. 1-14

ISSN: 2519-9889

Impact Factor: 3.426

medium is chosen while considering the solubility of the actives. Analytical methods can be used to analyze samples from the dissolving media.

\section{Microscopy studies ${ }^{[6]}$}

The microscopic aspects of the drug, nanosponges, and the result (drug/nanosponge complex) can be studied using scanning electron microscopy (SEM) and transmission electron microscopy (TEM). The production of complex formation is shown by the difference in crystallization states of the raw materials and the finished product as observed under an electron microscope.

\section{Drug release kinetics ${ }^{[2]}$}

The release data was analyzed using Zero order, First order, Higuchi, Korsemeyer-Peppas, Hixon Crowell, Kopcha, and Makoid-Banakar models to examine the mechanism of drug release from the Nanosponge. Graph pad prism software can be used to analyze the data. The software calculates the parameters of a nonlinear function that gives the best match between experimental data and the nonlinear function.

\section{Applications:}

Drugs tend to be incorporated into the structure of nanosponges, either as inclusion complexes or as non-inclusion complexes. Nanosponges have a wide range of potential applications in the pharmaceutical industry due to their biocompatibility and adaptability.

\section{Solubility enhancement ${ }^{[1][2][5]}$}

The low water solubility of many drugs is one of the most significant barriers to their development. About $40 \%$ of new drugs are water insoluble, which makes them difficult to use in clinical trials. The formulation of medications that are poorly water soluble is a tough problem to solve. Nanosponges can help compounds that have a low water solubility enhance their wetting and solubility. The medications can be molecularly disseminated within the nanosponge structure before being released as molecules, eliminating the need for dissolution. As a result, the drug's perceived solubility can be boosted. Many formulation and bioavailability issues can be overcome by increasing a substance's solubility and dissolving rate, and nanosponges can significantly improve drug solubility.

\section{Sustained delivery system ${ }^{[1]}$}

A modified-release product's design is often designed to help improve the treatment regimen by delivering the drug slowly and continuously over the dose period. This allows for a reduction in the dose given, a change in the pharmacokinetic profile, and a reduction in side effects. Using appropriate polymers and crosslinking agents, drug release kinetics from nanosponges can be achieved with a sustained release profile over time. Following encapsulation, nanosponges can be utilized to retain and extend the release of volatile compounds such as essential oils. 


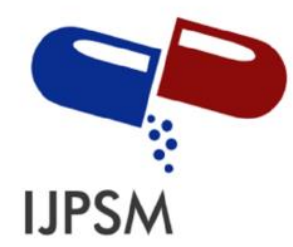

Shaikh Bilal J. et al, Int. Journal of Pharmaceutical Sciences and Medicine (IJPSM), Vol.6 Issue. 6, June- 2021, pg. 1-14

ISSN: 2519-9889

Impact Factor: 3.426

\section{Oral delivery systems ${ }^{[1]}$}

The rate at which a solid drug dissolves is a limiting factor for oral bioavailability. The dissolution process acts as a rate-controlling phase for hydrophobic drugs, determining the rate and degree of absorption. As a result, the absorption of many hydrophobic drugs from the gastrointestinal tract is irregular and partial. The complexes may be distributed in a matrix of excipients, diluents, and lubricants suitable for the production of capsules or tablets for oral delivery.

\section{Topical delivery systems ${ }^{[1]}$}

Conventional dermatological and personal care products often include active substances in high concentrations but for a short period of time. This could result in a cycle of short-term overmedication and long-term undermedication. When active substances permeate the skin, they might cause rashes or more significant side effects. This technique, on the other hand, provides for a consistent and consistent rate of release, reducing discomfort while preserving efficacy. Nanosponges can be used to suspend or entrap a wide range of components before being included into a manufactured product as a gel, lotion, cream, ointment, liquid, or powder. For topical application, nanosponges can be employed in gels or creams. The potential of nanosponges to boost solubility at the skin's surface can be linked to their ability to increase the uptake of the biomolecule by the skin.

\section{Protein delivery ${ }^{[1]}$}

A novel synthetic route was used to generate swellable cyclodextrin-based nanosponges for protein delivery. New swellable cyclodextrin-based poly (amidoamine) nanosponges (PAA-NS) were manufactured by cross-linking cyclodextrin with either 2,2-bis(acryl amidoacetic acid) or a short polyamido-amine chain derived from 2,2-bis(acryl amidoacetic acid) and 2-methylpiperazine, respectively. Using the high pressure-homogenization approach, PAANS was reduced in nanosuspensions. The $\mathrm{pH}$ of the surrounding media was found to affect the swellable nanosponges.

\section{Gas delivery ${ }^{[1]}$}

Nanosponge formulations can be used to store a variety of gases. The ability of -CD nanosponges to hold substantial volumes of carbon dioxide, 1-methylcyclopropene, and oxygen has been demonstrated. Many biomedical applications could benefit from the complexation of oxygen or carbon dioxide. The oxygen-filled nanosponges, in particular, could provide oxygen to hypoxic regions found in a variety of disorders. As oxygen encapsulating formulations, three distinct cyclodextrin nanosponges were produced by crosslinking $\alpha-, \beta$-, or -cyclodextrin with carbonyl diimidazole. Both in the presence and absence of ultrasound, the nanosponges were able to release oxygen (US). A nanosponge/hydrogel combination technique was used to achieve oxygen penetration across a silicone membrane. 


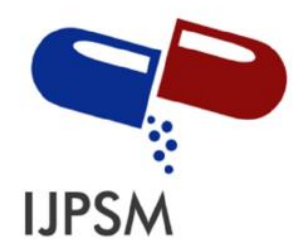

Shaikh Bilal J. et al, Int. Journal of Pharmaceutical Sciences and Medicine (IJPSM), Vol.6 Issue. 6, June- 2021, pg. 1-14

ISSN: 2519-9889

Impact Factor: $\mathbf{3 . 4 2 6}$

\section{Protection from light or degradation ${ }^{[1]}$}

Nanosponges can also be utilized as carriers to protect encapsulated molecules from degradation caused by light, chemicals, and enzymes. As a result, the molecule's stability and potency are improved.

\section{Removal of Organic Pollutants from Water ${ }^{[1][2]}$}

$\beta$-cyclodextrin nanosponges, which are completely water insoluble, have the ability to encapsulate organic pollutants in water. These Nanosponges can be injected into ceramic porous filters, resulting in hybrid organic/inorganic filter modules. These hybrid filter modules were put to the test for effective water purification using a variety of water pollutants. PAHs (polycyclic aromatic hydrocarbons) have been proven to be particularly effective in being eliminated (more than $95 \%$ ). Trihalogenmethanes (THMs), monoaromatic hydrocarbons (BTX), and pesticides (simazine) are among the pollutants that can be eliminated (>80\%).

\section{Nanosponges for cancer therapy ${ }^{[3]}$}

Because of their limited solubility, anticancer drug distribution is one of the most challenging tasks in the pharmaceutical industry today. According to one article, the nanosponge complex is three times more efficient than direct injection in reducing tumour growth. The complex of the nanosponge loads a medicine and exposes a targeting peptide that binds strongly to the tumour receptor's radiation-induced cell top layer. When nanosponges come into contact with a tumour cell, they stick to its surface and begin to release medication molecules. Targeting drug delivery has the advantage of providing a more effective therapeutic impact at the same dose with fewer side effects.

\section{As absorbent in treating poison in blood ${ }^{[3]}$}

By absorbing the toxin, nanosponges can remove the hazardous harmful chemical from our blood. Instead of utilizing antidotes, we can use nanosponges injected into the bloodstream to absorb the toxins. The nanosponge imitates a red blood cell in the bloodstream, fooling toxins into attacking it and then absorbing it. The toxin determines how many poison molecules each nanosponge can absorb.

\section{Nanosponges in enzyme immobilization ${ }^{[6]}$}

Immobilization of enzymes is particularly significant for lipases since it improves their stability and has an impact on properties such as enantio selectivity and reaction kinetics. As a result, novel solid supports that are suitable for this family of enzymes are constantly in demand. Pseudomonas fluorescens lipase adsorbed on a new type of cyclodextrin-based nanosponges demonstrated high catalytic performance, according to Boscolo et al.

\section{Oxygen Delivery System ${ }^{[4]}$}

These are characterized by the use of $\alpha, \beta$ and $\gamma$-cyclodextrins, which are suspended in water and get saturated with it. With the help of a nanosponge/hydrogel system, a silicone kind of membrane can also be used for oxygen permeation. They can also utilize it to treat hypoxic tissues that are caused by a variety of illnesses. 


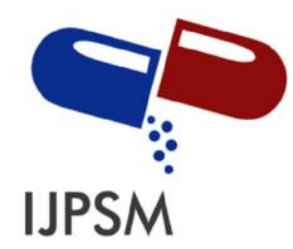

Shaikh Bilal J. et al, Int. Journal of Pharmaceutical Sciences and Medicine (IJPSM), Vol.6 Issue. 6, June- 2021, pg. 1-14

ISSN: 2519-9889

Impact Factor: 3.426

\section{Biomedical applications ${ }^{[15]}$}

Both hospitals and the medical industry depend primarily on oxygen, which can be difficult to store at times. Carbonate nanosponges based on cyclodextrin were designed to form inclusion complexes with several gases such as carbon dioxide, methylcyclopropene, and oxygen in a novel attempt to overcome this problem. These oxygencarrying nanosponges can be utilized to provide oxygen in hypoxic conditions.

\section{To provide stability ${ }^{[15]}$}

Nanosponges have the ability to selectively trap a few families of protein molecules from the blood, allowing them to be protected from enzymatic breakdown.

\section{As a carrier for calcium delivery ${ }^{[15]}$}

Most commercially available phosphate binders have a range of side effects, including bone disease, aluminium toxicity, soft tissue calcification, and hypercalcemia. Enteric-coated cyclodextrin-based calcium carbonate nanosponges that could bind efficiently to free phosphate ions and release calcium in a regulated way were developed and described by Pravin Shende et al. The cross-linking, according to the paper, improves the stability and help in the controlled release of calcium. All of these factors make it excellent for treating hyperphosphatemia without causing any negative side effects.

\section{Ocular delivery ${ }^{[15]}$}

Glaucoma is a long-term condition that increases the chance of eyesight loss. There are several approaches for increasing patient compliance, however poor adherence to these drugs is a serious barrier. Lambert et al. investigated the efficiency of nanosponges encapsulated chemicals in glaucoma therapy, determining that one injection of nanosponges may successfully deliver ocular antihypertensive molecules in a continuous, linear form for around 32 days. They even claimed that these formulations are effective in targeting degenerating retinal ganglion cells (RGC) in glaucoma sufferers.

\section{In detoxification and reduction of superbug infection ${ }^{[15]}$}

Antisera, monoclonal antibodies, small molecule inhibitors, and other current detoxification platforms work by targeting toxins based on their chemical structures. Che-Ming J. Hu et al. developed biomimetic toxin nanosponges that may operate as a toxin decoy in vivo, recognizing the need for tailored treatment for various diseases. These nanosponges were discovered to be capable of absorbing poisons that caused membrane damage. They could redirect toxins away from cellular sites after absorption, avoiding toxin-mediated hemolysis. This biologically inspired toxin nanosponges, according to the study, can be utilized to treat a wide range of ailments and injuries caused by pore-producing toxins, which are the most extensively identified protein toxins in nature to far. They even found that these nanosponges could significantly diminish the noxious effects of staphylococcal-hemolysin, suggesting that this strategy could be utilized to counter drug-resistant contaminations/infections like methicillin- 


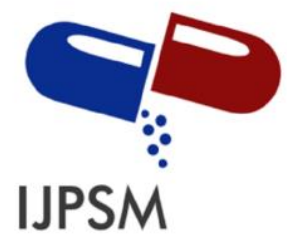

Shaikh Bilal J. et al, Int. Journal of Pharmaceutical Sciences and Medicine (IJPSM), Vol.6 Issue. 6, June- 2021, pg. 1-14

ISSN: 2519-9889

Impact Factor: 3.426

resistant Staphylococcus aureus infections. They claim that one RBC membrane can cover more than or about 3,000 of these stealthy nanosponges (completely loaded with poison) that can be safely eliminated via the liver.

\section{Nanosponges for drug delivery ${ }^{[2][3][4]}$}

The nanosponges are solid in nature and can be used in dosage forms such as oral, parenteral, topical, and inhalation. The complexes may be distributed in a matrix of excipients, diluents, lubricants, and anti-caking agents suitable for the formulation of capsules or tablets for oral delivery. The complex can be delivered in sterile water, saline, or other aqueous solutions for parenteral delivery. They can be efficiently integrated into topical hydrogels for topical delivery.

\section{Conclusion}

The difficulties of achieving extended-release in topical dosage forms in conventional dosage forms prompted the development of nanosponge technology. Ineffective formulation is one of the reasons why the majority of medication therapies fail. Because they can accommodate both hydrophilic and hydrophobic drugs, the development of nanosponge has become an important step in solving some challenges such as drug toxicity, low bioavailability, and predictable drug release. The advantages outnumber the numerous disadvantages connected with this formulation. A important criterion is the molecular weight of the component to be integrated into the formulation. However, further research is anticipated to overcome this obstacle. Nanosponges are a novel type of biocompatible cross-linked polymer with a flexible and cost-effective manufacturing method. This method allows substances to be entrapped, which reduces adverse effects, improves stability, and enhances elegance. Aside from drug delivery, they have potential use in cosmetics, biomedicine, bioremediation processes, agrochemistry, and catalysis, among others. Drugs supplied via nanosponges can be proven to be safe and effective, and clinical studies can demonstrate their potential for human use, which will assist the pharmaceutical industry tremendously.

\section{References}

[1]. Arshad Khan, Ahmed Khan, Eranti Bhargav; Nanosponges: A New Approach for Drug Targetting; International Journal of Pharmacy and Pharmaceutical Research 2016; 7(3); 381-396.

[2]. Shrishail M Ghurghure, MahewashPathan, Priyanka R Surwase; Nanosponges: A Novel approach for targeted drug delivery system; International Journal of Chemistry Studies; November 2018; 2(6); 15-23

[3]. Himangshu Bhowmik, D. NagasamyVenkatesh, AnuttamKuila, Kammari Harish Kumar; Nanosponges: A Review; International Journal of Applied Pharmaceutics 2018; 10(4); 1 - 5 


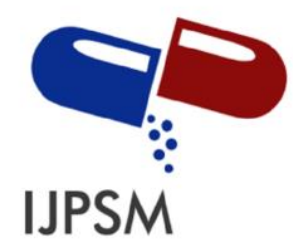

Shaikh Bilal J. et al, Int. Journal of Pharmaceutical Sciences and Medicine (IJPSM), Vol.6 Issue. 6, June- 2021, pg. 1-14

ISSN: 2519-9889

Impact Factor: 3.426

[4]. Vyas SP, Khar RK. Targeted and Controlled Drug Delivery- Novel Carrier Systems. Molecular Basis of Targeted Drug Delivery. CBS Publishers and Distributors. New Delhi. 2008: 38- 40.

[5]. Shubham Shrestha and Sankha Bhattacharya, Versatile Use of Nanosponge in the Pharmaceutical Arena: A Mini-Review; Recent Patents on Nanotechnology, 2020; 14.

[6]. AnuradhaSalunkhe, Seemadevi Kadam, SayaliMagar and KiranDangare; Nanosponges: A Modern Formulation Approach In Drug Delivery System; World Journal of Pharmacy and Pharmaceutical Sciences; 7(2); 575-592.

[7]. K. Muni Raja Lakshmi, CH. Suma Shree, N. Lakshmi Priya;Nano Sponges: A Novel Approach for Targeted Drug Delivery Systems; Journal of Drug Delivery and Therapeutics; 2021; 11(2): 247-252.

[8]. Simranjot Kaur, Sandeep Kumar; Nanosponges: An Innovative Drug Delivery System; Asian Journal Pharmceutical and Clinical Research 2019; 12(7); 60-67.

[9]. Prashant D. Jain, Monika L. Girase, Krunal B. Borse, Yash K. Karandikar, Sanket S. Chintawar, Ayush S. Kharache, Adarsh Mohan Gupta;"nanosponge: a versatile drug delivery system”- review;April 2019|1(11); $110-115$.

[10].Tarun Kumar Satpathy, NeeleshChaubey, B. Usha Sri and Venkata R. Naidu; Nanosponges - Novel Emerging Drug Delivery System; International Journal Of Pharmaceutical Science And Research; Page No. 1 - 10.

[11].Shastrulagari Shivani and Kranthi Kumar Poladi; Nanosponges - Novel Emerging Drug Delivery System: A Review; International Journal Of Pharmaceutical Science And Research; Page No. 1 - 11.

[12].https://en.wikipedia.org/wiki/Nanosponges

[13].Ajay Vishwakarma, PreetamNikam, Rajendra Mogal, Swati Talele; Review On Nanosponges: A Benefication For Novel Drug Delivery; International Journal of PharmTech Research; Jan-March 2014; 6(1); 11-20.

[14].ShashikantSudarshanUpadhye, Dr. SrinathBalkundhi, Dr. VishwajeetSampatraoGhorpade, Dr. Shirishkumar Damodar Ambavade, Mr. SujitVitthalAbhang, Ms. Safina Ismail Mulla, Mrs. PriyaAbhishek Patil; Nanosponges: An Innovative Approach for Targeted Drug Delivery System; Research Journal of Pharmacy and Technology 2021; 14(3); 1 -21.

[15].Pavithra Pradeep Prabhu, ChetanHasmukh Mehta, Usha Y Nayak; Nanosponges -Revolutionary Approach: A Review; Research Journal of Pharmacy and Technology 2020; 13(7); 1 - 9. 